\title{
MODELLING AND SIMULATION OF LATTICE STRUCTURES USING VARIOUS MATERIAL MODELS FOR POLYMERIC MATERIALS
}

\author{
Kerstin Lehner*, Anna Kalteis, Zoltan Major \\ Institute of Polymer Product Engineering, Johannes Kepler University Linz, Altenbergerstraße 69, 4040 Linz, \\ Austria \\ * corresponding author: kerstin.lehner@jku.at
}

\begin{abstract}
Based on lightweight design concepts, lattices are increasingly considered as internal structures. This work deals with the simulation of periodically constructed lattices to characterize their behaviour under different loadings considering various material models. A thermo-mechanical analysis was done, which is resulting in negative CLTE-values (Coefficient of Linear Thermal Expansion). Simulations with linear-elastic behaviour were evaluated regarding the tensile, compression and shear modulus and the Poisson's ratio. Some of the investigated structures behave auxetic. Beside the linear elastic behaviour, also the hyper-elastic and visco-elastic behaviour of some structures were investigated. Furthermore, elasto-plastic simulations were performed where the applied loading was biaxial. As a result the initial yield surfaces were presented. The individual RVEs (Representative Volume Elements) can be utilized for different areas of application dependent on the used materials.
\end{abstract}

KEYWORDS: periodic lattice structures, material models, auxetic behaviour, negative CLTE.

\section{INTRODUCTION}

Based on lightweight design concepts, lattices are increasingly considered for additively manufactured components as internal structures in spite of classical bulk solid volumes. The structured construction of cellular materials has extremely advantageous properties for the lightweight design represented by a low material density combined with high stiffness and strength as well as an excellent damping behaviour [1. Dependent on the desired applications, various materials possessing different behavioural mechanisms are utilized for the design of components. The aim of the present work was to characterize and compare various modelled regular open cellular materials defined as lattice structures with applied periodic boundary conditions regarding different material models under various loading conditions. Fig, 1, depicts the task and procedure of the work performed. The results of the structures which are presented in this paper are highlighted. These results are chosen because they are the most informative ones. Linear-elastic material behaviour is the simplest assumption of elasticity and occurs for small deformations [2]. Thermoplastic elastomers behave hyper-elastically, which is characterized by a non-linear deformation behaviour and a unique stored strain energy density function depending on the current strain [3]. Visco-elastic materials behave both viscous and elastic and show time-, temperature- and frequency-dependent characteristics 4. Elasto-plasticity describes polymeric materials, in which plastic deformation occurs when loaded beyond its limit of linear-elastic behaviour. In the next section the simulation approaches and results are presented and in the last section a conclusion for various lattice structures and different material models is drawn.

\section{Methodology}

In the following the methodology is described how the lattice structures are modelled and simulated. The structures are modelled in the software NX, which was developed by the company Siemens. It is an interactive CAD/CAM/ CAE-system. Fig/2 shows the procedure of the modelling of the various structures represented by an Octet structure. A matrix block is modelled by extrusion of a square with the dimensions $50 \times 50 \mathrm{~mm}^{2}$. Within this assembly space sketches are drawn along the desired geometry of the trusses. These are fully parametrised, which is when the size of the Representative Volume Element (RVE) is changed the inclusion size is automatically adapted. At these curves filled tubes are positioned, which are connected to each other. Boolean operations were applied to first intersect the protruded inclusion parts from the RVE and then subtract the inclusion from the matrix to get two individual parts. With these structures finite element simulations are performed in the software Abaqus CAE (Simulia, Dassault Systemes). In order to model an RVE in such a way that there is no influence of edge effects, which means that the RVE has to be modelled as if being part of the continuous bulk material, commonly so called PBCs are used. Using PBCs the single RVE behaves as if being part of a continuum material [5]. Therefore, Perodic Boundary Conditions (PBCs) are implemented in Abaqus CAE by a Python script, which was developed at the Institute of Polymer Product Engineering (IPPE) by 


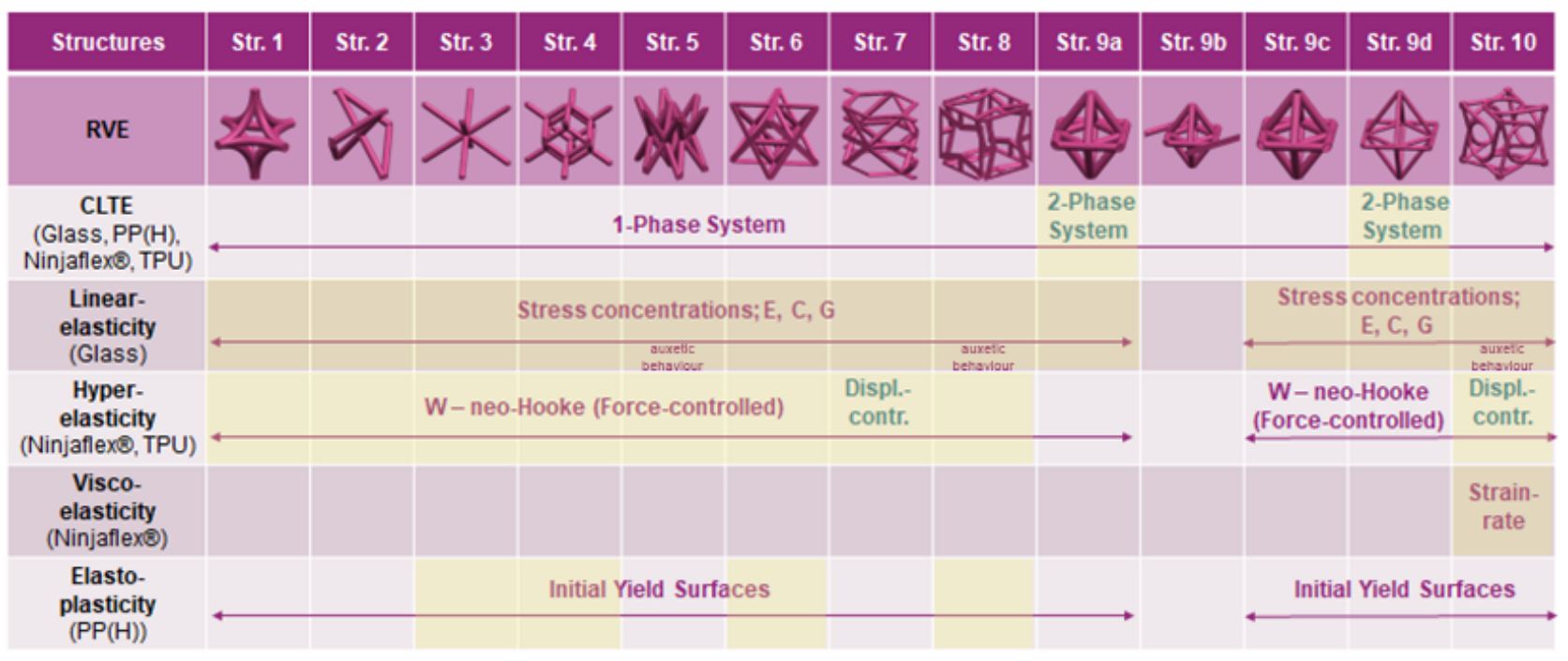

FiguRE 1. Task and procedure of the present work.

Dipl.-Ing. Dr. Matei-Constantin Miron and Dipl.Ing. Emil Jacob Pitz. The specific procedure and mechanism of this script can be found in 6 .

\section{THERMO-MECHANICAL ANALYSIS}

Thermal expansion is the tendency of matter to change in shape, area and volume in response to a change in temperature [7]. In the case of linear observation, the one-dimensional change in length is discussed. The strain in the direction of the length, which is equally to the relative expansion, divided by the change in temperature is called the material's Coefficient of Linear Thermal Expansion (CLTE) and varies with temperature for most materials. The mathematical formula for the CLTE $\alpha$ is given in Eq1 $\Delta L$ is the change in length $\left(\Delta L=L_{1}-L_{0}\right), L_{0}$ is the initial length of the specimen and $\Delta T=T_{1}-T_{0}$ represents the temperature change.

$$
\alpha=\frac{\Delta L}{L_{0}} \cdot \frac{1}{\Delta T}
$$

For CLTE-simulations predefined fields with a constant temperature rise of $10 \mathrm{~K}$ throughout the whole region are implemented. The reference points for the three directions in space, which are created by a script for periodic boundary conditions and mark the centres of the adjacent unit cells, are set free to permit expansion in all directions. The partitioning of Structure 9 for the two-phase system is given in Fig 3 with the outer part coloured grey and the inner part coloured purple. Two different variations of Structure 9 are investigated: Structure 9a (tube diameter of $5 \mathrm{~mm}$ ) and Structure $9 \mathrm{~d}$ (tube diameter of $2.5 \mathrm{~mm}$ ). The used material data for the thermo-mechanical simulations are given in Tab.1. Material 1 has a lower E-Modulus, a lower $\alpha$ and a higher $\nu$ than Material 2. The resulting von Mises stresses can be seen in Fig.4 (top) for a material composition where the inner square is made

\begin{tabular}{lll}
\hline & Material 1 & Material 2 \\
\hline$E, \mathrm{MPa}$ & 1000 & 10000 \\
\hline$\nu,-$ & 0.45 & 0.2 \\
\hline$\alpha, \frac{1}{K}$ & $1 \mathrm{E}-6$ & $1 \mathrm{E}-5$
\end{tabular}

TABLE 1. Material data for CLTE-simulations [8].

of a stiffer material with a higher Coefficient of Linear Thermal Expansion (Material 2) than the outer structural part. The maximum stresses occur at the connection points because of the hindered expansion of the inner square due to the lower expansion of the outer parts. Fig. 4 (bottom) shows the reversed construction where the inner square consists of Material 1. Greater stresses occur in the outer geometry part. When using Material 1 for the outer structural part the CLTEs yield in negative values for the two variations of Structure 9 and therefore contraction in z-direction (Fig. 5 1. and 2.). This effect is traced to a mechanical contraction normal to the plane in which the inner square consisting of Material 2 lies resulting from the higher expansion of the inner material. This mechanical contraction overcomes the expansion due to the temperature increase and therefore yields to a negative CLTE in z-direction for this structure. Compared to the literature results of $[8$, this lattice structure reveals nearly the same value of the thermal expansion in z-direction. When the outer structural part is made out of Material 2 and the inner square consists of Material 1 both investigated structures expand when increasing the temperature (Fig. 5. 3. and 4.). For this material composition the highest CLTE-values are received in $\mathrm{z}$-direction.

\section{LINEAR-ELASTICITY}

Linear-elasticity describes the material behaviour at small deformations [2]. Most elastic materials exhibit 


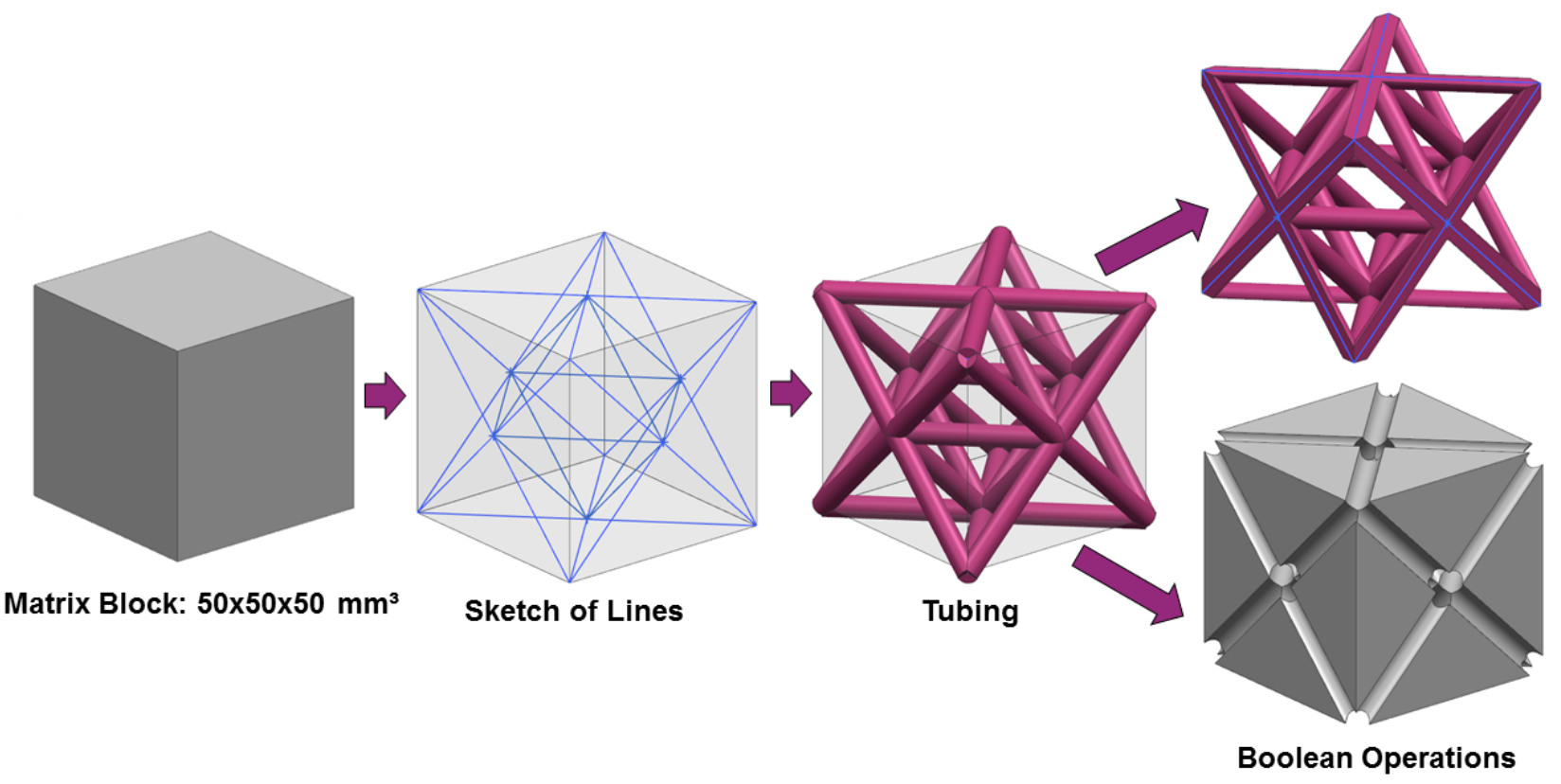

Figure 2. Modelling procedure represented by an Octet structure.

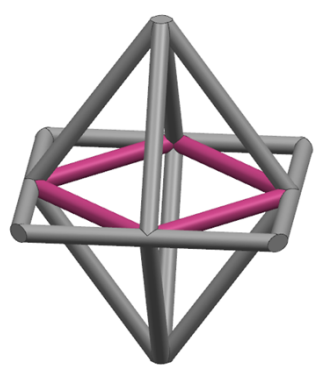

Figure 3. Partitioning of Structure 9.

a linear-elastic model, which is time-independent, and can be described by a linear relation between the stress $\sigma$ and strain $\epsilon$. Linear-elastic behaviour for the lattice structures is characterized by the apparent lattice stiffness matrix wherefore displacement-controlled simulations of uniaxial tension $\left(E_{T}\right)$ and shearing $(G)$ were performed in all directions to determine the entries of the stiffness matrix. For further investigations of the linear behaviour also simulations of uniaxial compression $\left(E_{C}\right)$ were done. The following input parameters are used for the linear-elastic simulations: $E=72000 M P a, \nu=0.2$. In Fig, 6 it can be seen that Structure 6 shows the stiffest behaviour of all investigated structures in all directions. Structure 3 has the lowest stiffness values related to tension and compression, but this RVE shows a good applicability for purposes where shear loads are present. When comparing the different variations of Structure 9 it can be seen that Structure 9a (tube diameter of $5 \mathrm{~mm}$ ) results in the highest stiffness values whilst Structure 9c (hollow tubes with a shell thickness of $0.5 \mathrm{~mm}$ )
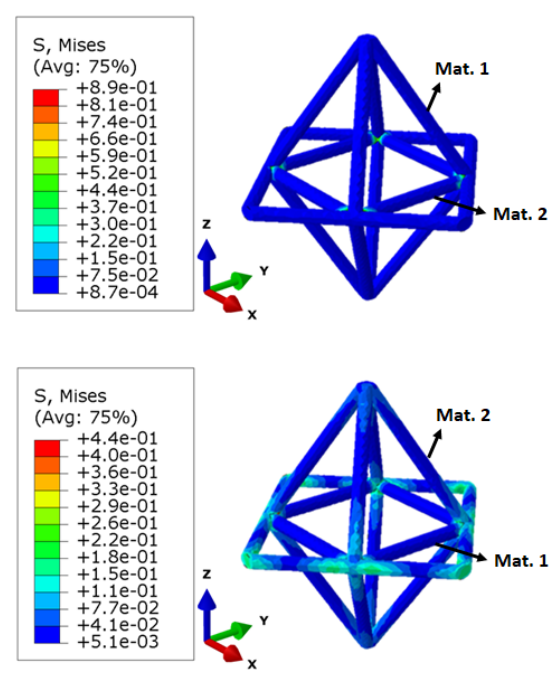

Figure 4. Von Mises stresses of a two-phase system caused by temperature increase.

and Structure 9d (tube diameter of $2.5 \mathrm{~mm}$ ) exhibit lower values, but also have the maxima in z-direction. Special characteristics of the Poisson's ratio can be observed for Structure 5 (Fig.7](top)), Structure 8 (Fig.7 (bottom left)) and Structure 10 (Fig 7 (bottom right)). In Fig.7 these structures are illustrated and the von Mises stress is depicted for different loading directions. Structure 5 expands in y-direction when pulling in $\mathrm{x}$-direction and vice versa, which is described by negative values for $\nu_{12}$ and $\nu_{21}$ (auxetic behaviour). With tension in $\mathrm{z}$-direction this structure bears very high strains in $\mathrm{x}$ - and $\mathrm{y}$-direction. Structure 8 shows an overall auxetic behaviour. Due to the twisting mo- 


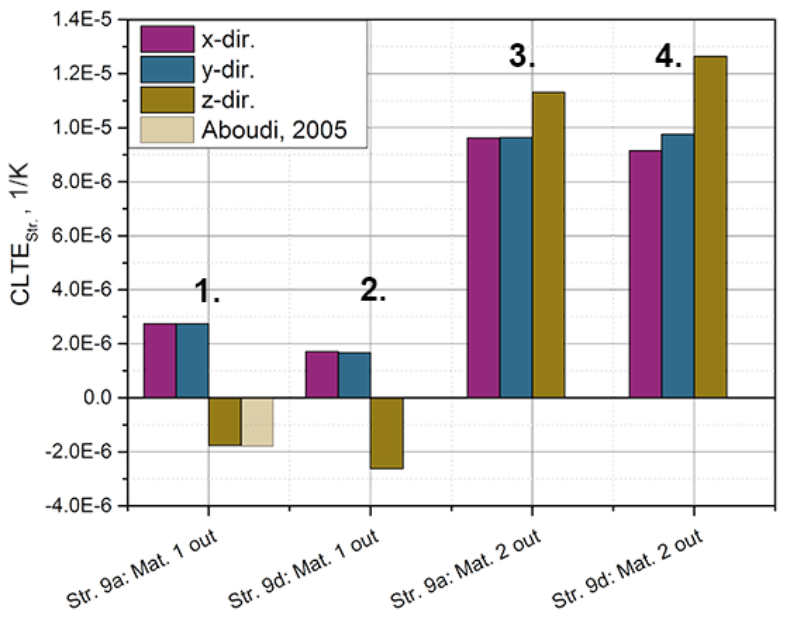

Figure 5. Von Mises stresses of a two-phase system caused by temperature increase.

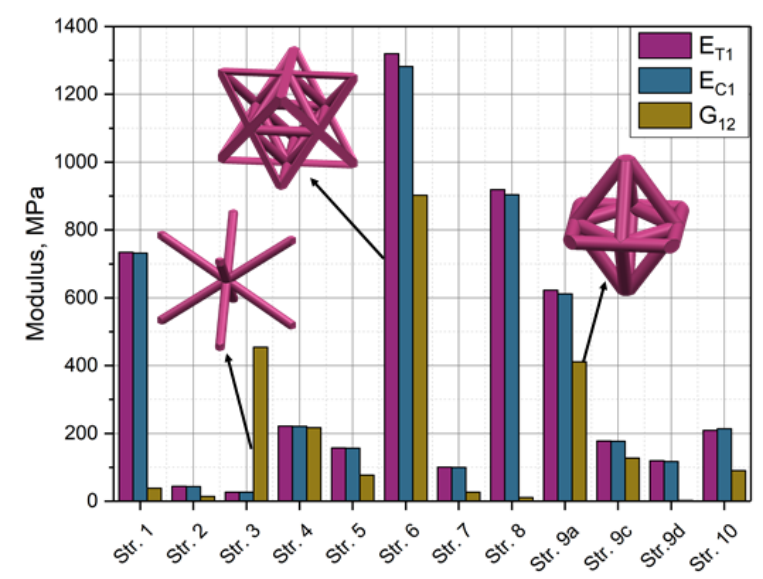

Figure 6. Comparison of the different structures regarding tensile $\left(E_{T 1}\right)$, compression $\left(E_{C 1}\right)$ and shear $\left(G_{12}\right)$ modulus.

tion, Structure 10 also behaves auxetic and therefore exhibits negative values for the Poisson's ratios.

\section{HYPER-ELASTICITY}

Hyper-elasticity represents a non-linear, isotropic and time-independent form of the elasticity. The relationship between an applied stress and the strain is linear for very small strains, but will reach a plateau in the stress-strain curve at a certain point 3. The Strain Energy Density Function W is evaluated using the displacements resulting from the forcecontrolled simulations according to the law of NeoHookean solids, which is presented in Eq.2 where $C_{10}$ is a material constant and $I_{1}$ is the first invariant of the stress tensor. The hyper-elastic material model (Neo-Hooke) was generated from experimental stress-strain-data resulted from uniaxial tensile tests of the bulk materials Ninjaflex ${ }^{\circledR}(E=6.5 \mathrm{MPa}$, $\nu=0.495)$ and TPU3 $(E=9.5 M P a, \nu=0.495)$. The material constant $C_{10}$ for the Neo-Hookean model

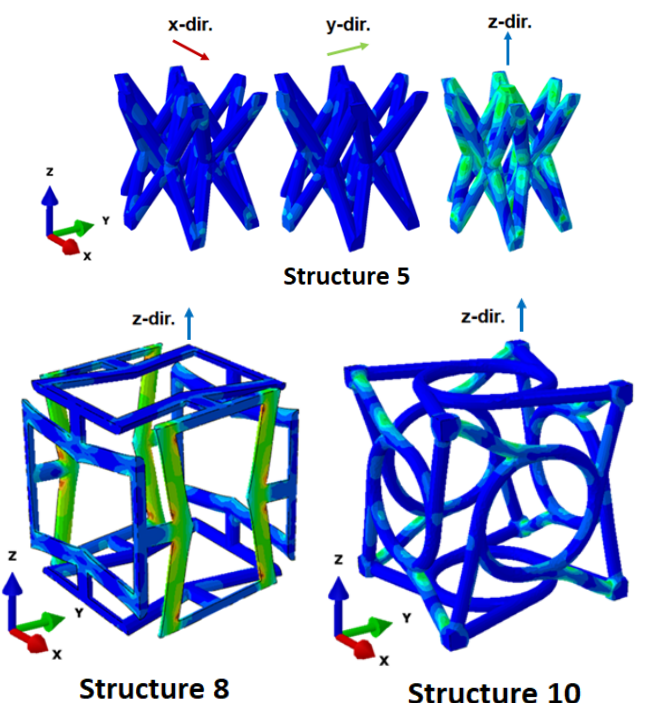

FigURE 7 . Illustration of the auxetic behaviour of Structure 5, 8 and 10 .

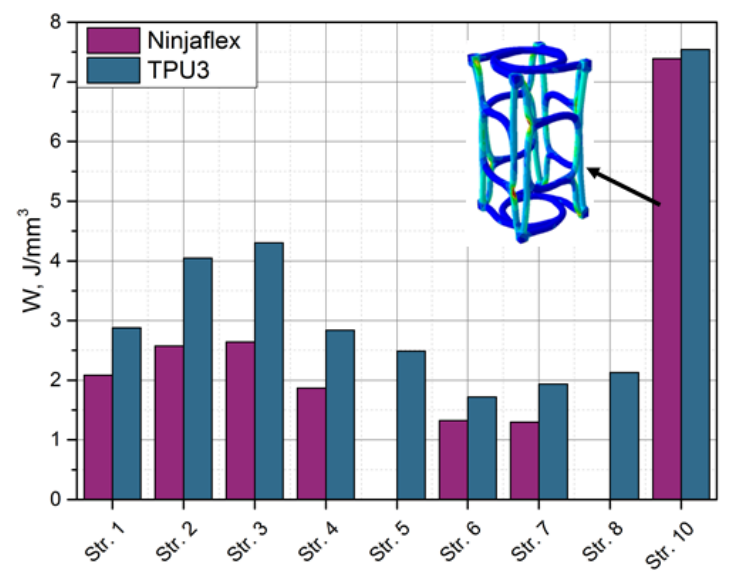

Figure 8. Comparison of $\mathrm{W}$ for the different lattice structures and for the two materials.

has the value $C_{10}=0.78057 \frac{\mathrm{N}}{\mathrm{mm}^{2}}$ for Ninjaflex ${ }^{\circledR}$ and $C_{10}=1.56371 \frac{\mathrm{N}}{\mathrm{mm}^{2}}$ for TPU3.

$$
W_{N H}=C_{10} \cdot\left(I_{1}-3\right)
$$

A bar chart of the $\mathrm{W}$-values of the various relevant structures is given in Fig,8. It can be seen that Structure 10 reveals the highest values for $\mathrm{W}$, which means that the applied force can be stored in the material as an energy to change the shape of the structure. Therefore, this structure is the best fitting structure of all investigated RVEs for hyper-elastic characteristics.

\section{ViSCO-ELASTICITY}

Visco-elastic materials combine the characteristics of solids and liquids, and thus behave in part elastically and in part plastically. The effect of visco-elasticity is time-, temperature- and frequency-dependent. To determine the visco-elastic material behaviour two strainrates were evaluated. The simulations were performed 
dynamic, implicit to allow strain-rate dependent calculations. The applied loadings were $0.5 \frac{\mathrm{mm}}{\mathrm{s}}$ and $5 \frac{\mathrm{mm}}{\mathrm{s}}$ for uniaxial tension. For the visco-elastic simulations experimental data from a Dynamic-Mechanical Thermal Analysis (DMTA) were adapted into master curves with the help of the program ViscoShift (Ingenieurbuero Herdy) and the Prony parameters (Tab,2 were calculated in the program ViscoData (Ingenieurbuero Herdy). The directly calculated parameters $e_{i, \text { Prony }}, k_{i, \text { Prony }}$ and $\tau_{i, \text { Prony }}$ are representing the Young's modulus, the compression modulus and the relaxation time, while the shear modulus $g_{i, \text { Prony }}$ has to be calculated by $g_{i, \text { Prony }}=\frac{e_{i, \text { Prony }}}{2 \cdot(1+\nu)}$ when assuming linear visco-elastic behaviour. The details of the exact implementation of the visco-elastic material behaviour into Abaqus can be found in 9. In order to be able to compare the results, these simulations were performed for Structure 10 as well as for a multipurpose tensile specimen according to ISO 527-1. In Fig.9 it can be seen that an increased strain-rate leads to a stiffer behaviour for the investigated material independent of the structure. When comparing the two specimens the results show lower stress values for Structure 10 (Fig 9 (top)) than for the multi-purpose tensile specimen (Fig 9 (bottom)), which represents the bulk material. This effect is traced to the low relative density of the lattice structure.

\section{Elasto-Plasticity}

In real media, deformation is linearly elastic only up to a certain limit. If this limit is exceeded and the material is therefore loaded beyond its yield stress, in ductile materials plastic deformation occurs 8. After reaching the Yield point, which is the point on the stress-strain curve that indicates the limit of elastic behaviour and the beginning of plastic behaviour, the stressed body does not return to its original form when it gets unloaded. For the characterization of elastoplastic material behaviour the initial yield surfaces were determined. Therefore simulations of biaxial loading conditions and coupled tension/compressionsimulations were necessary. The beginning of plasticity is assumed where a deviation of the experimental curve from linear behaviour can be detected to represent the real curve progression. When considering the physical yield point at approximately $32 \mathrm{MPa}$ the simulation model would be linear up to this point and would therefore not be suitable as approximation for the elasto-plastic behaviour. Hence, the plastic strain and the yield stress for $\operatorname{PP}(\mathrm{H})(E=1990 M P a$, $\nu=0.42)$, which represent elasto-plastic material behaviour, are shown in Fig, 10 (Yield point) and implemented in Abaqus using Mises-plasticity without consideration of triaxiality. In Fig 11 the RVEs are pictured on the left side showing the locations of the beginning of yielding in red for biaxial tension in $y$ - and z-direction. The blue colour indicates elastic behaviour. On the right side the yield points for

\section{Structure 10}

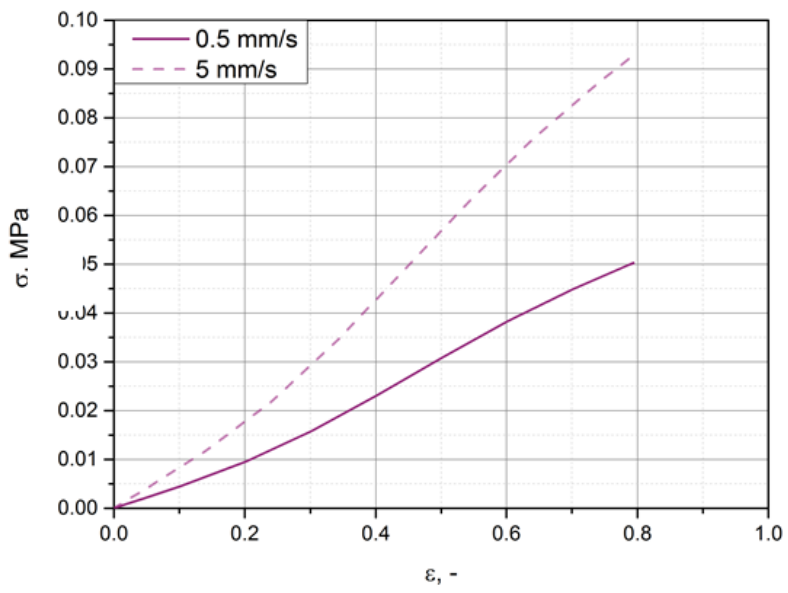

ISO-MPS

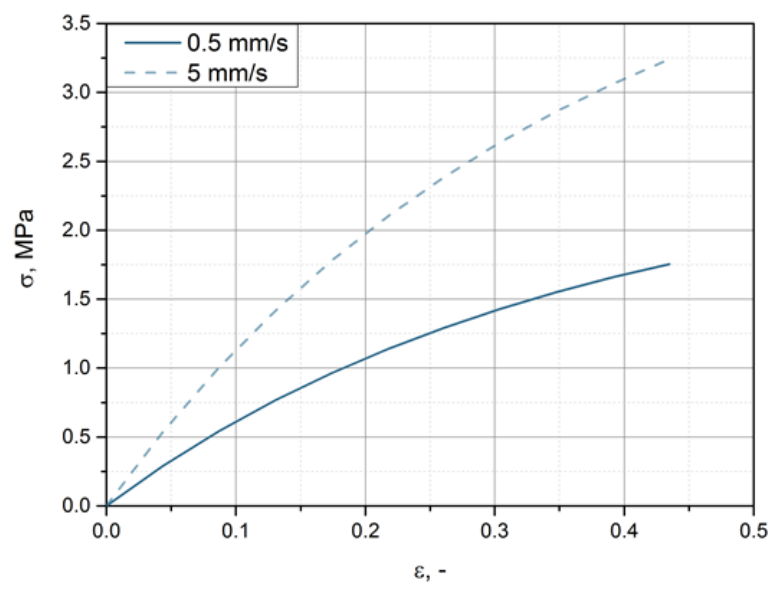

FigURE 9. Illustration of visco-elastic behaviour of Str. 10 (top) and ISO-MPS (bottom).

the different load cases are connected with straight lines. These lines mark the border between elastic and plastic behaviour. If the stresses exceed this border, plasticity is present. Additionally, the initial yield surfaces resulting from the yield criteria of von Mises and Tresca are plotted. The Tresca criterion (Eq.3) is marked with a dotted line and the von Mises criterion $(\mathrm{Eq}, 4)$ is drawn as a dashed line.

$$
\begin{aligned}
\sigma_{T}= & \max \left\{\frac{1}{2}\left|\sigma_{1}-\sigma_{2}\right|, \frac{1}{2}\left|\sigma_{2}-\sigma_{3}\right|, \frac{1}{2}\left|\sigma_{3}-\sigma_{1}\right|\right\} \\
\sigma_{v}^{2} & =\frac{1}{2} \cdot\left[\left(\sigma_{11}-\sigma_{22}\right)^{2}+\left(\sigma_{22}-\sigma_{33}\right)^{2}\right. \\
& \left.+\left(\sigma_{33}-\sigma_{11}\right)^{2}+6 \cdot\left(\sigma_{23}^{2}+\sigma_{31}^{2}+\sigma_{12}^{2}\right)\right]
\end{aligned}
$$

The initial yielding of Structure 3 (Fig 11(top)) takes place in its centre and therefore possesses lower yield stresses than Structure 4 (Fig 11(top centre)), the centre of which is separated into more connection points. Structure 8 (Fig,11(bottom)) shows the highest stress values for the transition between elasticity and plasticity for biaxial loading with tension in one 


\begin{tabular}{cccc}
\hline$e_{i, \text { Prony }},-$ & $g_{i, \text { Prony }},-$ & $k_{i, \text { Prony }},-$ & $\tau_{i, \text { Prony }}, s$ \\
\hline $2.38 \cdot 10^{-18}$ & $7.96 \cdot 10^{-19}$ & $7.93 \cdot 10^{-17}$ & $1.29 \cdot 10^{-03}$ \\
$1.40 \cdot 10^{-18}$ & $4.68 \cdot 10^{-19}$ & $4.67 \cdot 10^{-17}$ & $2.98 \cdot 10^{-02}$ \\
$6.44 \cdot 10^{-18}$ & $2.15 \cdot 10^{-18}$ & $2.15 \cdot 10^{-16}$ & $6.85 \cdot 10^{-01}$ \\
$1.27 \cdot 10^{-17}$ & $4.24 \cdot 10^{-18}$ & $4.22 \cdot 10^{-16}$ & $1.58 \cdot 10^{+01}$ \\
$8.33 \cdot 10^{-20}$ & $2.79 \cdot 10^{-20}$ & $2.78 \cdot 10^{-18}$ & $3.63 \cdot 10^{+02}$ \\
$2.49 \cdot 10^{-17}$ & $8.33 \cdot 10^{-18}$ & $8.30 \cdot 10^{-16}$ & $8.36 \cdot 10^{+03}$ \\
$6.92 \cdot 10^{-17}$ & $2.32 \cdot 10^{-17}$ & $2.31 \cdot 10^{-15}$ & $1.92 \cdot 10^{+05}$ \\
$3.97 \cdot 10^{-16}$ & $1.33 \cdot 10^{-16}$ & $1.32 \cdot 10^{-14}$ & $4.43 \cdot 10^{+06}$ \\
$2.11 \cdot 10^{-02}$ & $7.04 \cdot 10^{-03}$ & $7.02 \cdot 10^{-01}$ & $1.02 \cdot 10^{+08}$ \\
\hline
\end{tabular}

TABle 2. Prony parameters: Ninjaflex ${ }^{\circledR}$.

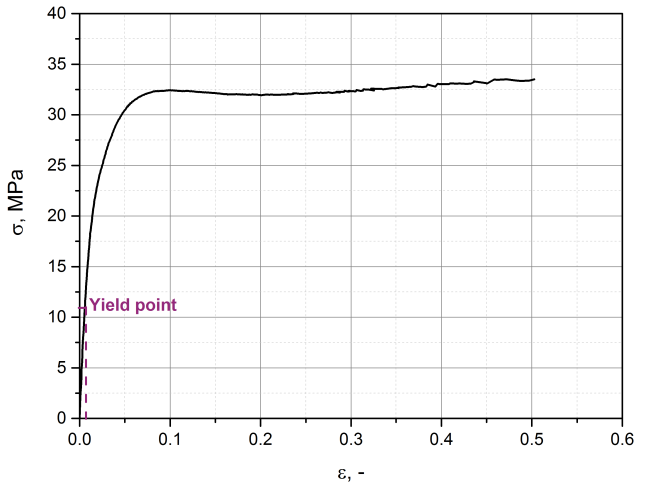

Figure 10. Experimental data for elasto-plastic material model: $\mathrm{PP}(\mathrm{H})$.

direction and simultaneous compression in the other direction, whereas all non-auxetic structures have the highest yield stresses for loadings with the same sign. For Structure 4 (Fig.11.(top centre)) the Tresca yield criterion is very similar to the resulting yield surface and for Structure 6 (Fig 11. (bottom centre)) the von Mises criterion would be a good approximation.

\section{Conclusion}

Performing a thermo-mechanical analysis simulations with partitioned inclusion geometries, which were applied with two different materials with various expansion behaviour, were conducted. The findings of [8] that a specific structural composition leads to a negative Coefficient of Linear Thermal Expansion could be validated for two-phase systems. Regarding the parameters of the apparent lattice stiffness matrix, Structure 6 was presented as the stiffest of all investigated structures in all directions using linear-elastic material behaviour. The lowest stiffness values related to tension and compression were detected for Structure 3. Negative values for the Poisson's ratio, which characterizes auxetic behaviour, could be observed for Structure 5, Structure 8 and Structure 10. After performing force-controlled simulations with hyperelastic material behaviour the Strain Energy Density Function was calculated according to the law of NeoHookean solids. Structure 10 revealed the highest values for $\mathrm{W}$, which made this RVE most suitable for large-strain non-linear elastic material behaviour.
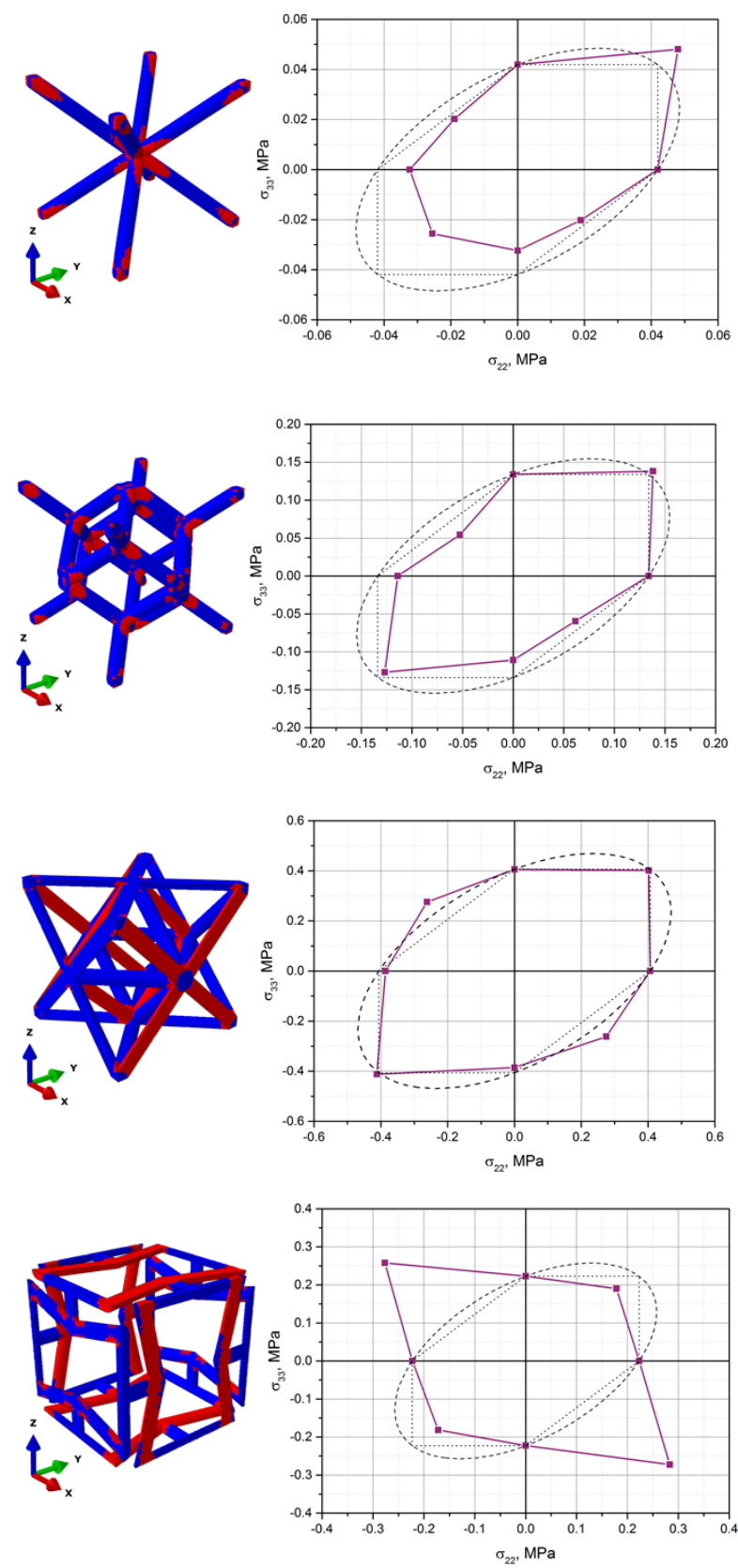

Figure 11. Initial Yield Surface: Str. 3, 4, 6, 8. 
Visco-elastic simulations were performed using Prony parameters of a generalized Maxwell model to investigate the strain rate dependency of Structure 10 compared to a multi-purpose tensile specimen. Whilst an increased strain-rate led to a stiffer behaviour for the investigated material independent of the structure, Structure 10 generally resulted in lower stress values than the bulk material. In order to represent the results of elasto-plastic material behaviour, initial yield surfaces were determined by applying biaxial loading conditions. In contrast to all investigated geometries, Structure 2 and Structure 3 exhibited the lowest yield stresses while Structure 5 and Structure 6 could withstand the highest stresses before yielding. All in all it was shown that the various lattice structures show different thermal and mechanical behaviour and therefore can be utilized for various purposes in different areas of application dependent on the used materials.

\section{REFERENCES}

[1] K. Drechsler, I. Karb, R. Kehrle, V. Witzel.

Faserverbundstrukturen, Leichtbau in Natur und

Technik. Themenheft Forschung Leichtbau pp. 14-21, 2007.

[2] P. Kelly. Solid Mechanics Part I: An Introduction to Solid Mechanics, chap. 6, pp. 145-153. 2013.
[3] M. Boyce, E. Arruda. Constitutive models of rubber elasticity: A review. Rubber Chemistry and Technology 73(3):504-523, 2000.

[4] M. Blanter, I. Golovin, H. Neuhäuser, H. Sinning. Internal friction in metallic materials, vol. 90. Springer-Verlag Berlin Heidelberg, 2007.

[5] V. D. Nguyen. Computational homogenization of cellular materials capturing micro-buckling, macro-localization and size effects. Ph.D. thesis, Universite de Liege - Computational and Multiscale Mechanics of Materials. 2014.

[6] E. J. Pitz. Multiscale Progressive Damage Modelling in Continuously Reinforced Composite Structures. Master thesis, Johannes Kepler University Linz, 2017.

[7] P. A. Tipler, G. Mosca. Physics for Scientists and Engineers, vol. 1. Macmillan Education, 2007.

[8] J. Aboudi, R. Gilat. Micromechanical analysis of lattice blocks. International Journal of Solids and Structures 42(15):4372-4392, 2005.

[9] M. Machado, U. Cakmak, I. Kallai, Z. Major. Thermomechanical viscoelastic analysis of woven-reinforced thermoplastic-matrix composites. Composite Structures 157:256-264, 2016. 